\title{
DETERMINAÇÃO DE GLICEROL LIVRE E TOTAL EM AMOSTRAS DE BIODIESEL POR MÉTODO ENZIMÁTICO COM DETECÇÃO COLORIMÉTRICA
}

\author{
Higor da Cal Valdez, Roberto Salgado Amado, Flávia Carvalho de Souza e Eliane D'Elia* \\ Instituto de Química, Universidade Federal do Rio de Janeiro, Av. Athos da Silveira Ramos, 149, Centro de Tecnologia, Bl. A, \\ Cidade Universitária, 21941-909 Rio de Janeiro - RJ, Brasil \\ Eduardo de Castro Vieira \\ CENPES/PETROBRAS, Av. Horacio Macedo 950, Cidade Universitária, 21941-915 Rio de Janeiro - RJ, Brasil
}

Recebido em 11/4/11; aceito em 20/9/11; publicado na web em 6/12/11

\begin{abstract}
FREE AND TOTAL GLYCEROL DETERMINATION IN BIODIESEL SAMPLES BY ENZYMATIC METHOD WITH COLORIMETRIC DETECTION. A method based on enzymatic activities was developed using three enzymes (glycerokinase, glycerol-3-phosphate oxidase and peroxidase) and colorimetric detection for the determination of glycerol in biodiesel. The enzymatic conversion of glycerol produces $\mathrm{H}_{2} \mathrm{O}_{2}$ that is eliminated by the action of peroxidase, an oxygen acceptor and 4- aminoantipirine, producing water and a colored compound, which was analyzed. This method showed good linear correlation coefficient $(\mathrm{r}=0.9937)$ in the concentration range of $4.95 \times 10^{-5}$ to $3.96 \times 10^{-4} \%(\mathrm{w} / \mathrm{w})$ and had experimental limits of detection and quantitation of $7.10 \times$ $10^{-6}$ and $2.10 \times 10^{-5 \%}(\mathrm{w} / \mathrm{w})$, respectively.
\end{abstract}

Keywords: glycerol content; biodiesel; enzymatic-colorimetric method.

\section{INTRODUÇÃO}

Atualmente, o biodiesel é encontrado misturado ao óleo diesel nos postos de abastecimento. O biodiesel é obtido a partir da reação de transesterificação de óleos vegetais com alcoóis primários, na presença de catalisador ácido ou básico. ${ }^{1}$

Esta reação produz um sistema bifásico com uma fase não polar de ésteres de ácidos graxos e outra mais densa constituída por glicerol e outros componentes residuais do processo. Após a decantação e separação, o biodiesel é purificado através de um processo de lavagem com água para retirar resíduos de glicerol livre e outros contaminantes.

A presença de glicerol livre (GL) e de glicerídeos, quantificados indiretamente pelo glicerol total (GT), no produto final pode, em níveis elevados, levar à formação de depósitos no motor, daí a necessidade de serem monitorados e controlados. ${ }^{2,3}$ Para tanto, a Agência Nacional do Petróleo, Gás Natural e Biocombustíveis (ANP) baixou a resolução $\mathrm{N}^{\circ} 4$ de 2010 onde limita o teor máximo de GL em 0,02\% e de GT em $0,25 \% \mathrm{~m} / \mathrm{m}^{4}$

Dentre todos os procedimentos disponíveis para análise de GL e GT, os métodos cromatográficos e enzimáticos oferecem os melhores desempenhos analíticos. Até o momento, a cromatografia a gás (CG) é a técnica recomendada pela ANP para a determinação de glicerol livre e total em biodiesel, pois apresenta boa precisão para a quantificação de componentes minoritários, podendo ser realizada em modalidade inteiramente automatizada, o que a torna o método preferido para aplicações industriais.

O primeiro trabalho sobre o uso de CG para a quantificação de ésteres totais, bem como de mono-, di- e triglicerídeos utilizou amostras de biodiesel de soja. As amostras foram tratadas com N,O-bis(trimetilsilil) trifluoracetamina (BSTFA) para converter grupamentos hidroxílicos em seus trimetilsilil derivados (TMS) correspondentes. Este tipo de transformação química foi o procedimento utilizado para a quantificação de glicerídeos em biodiesel por CG nos artigos subsequentes. ${ }^{5}$

Um método para análise de GL foi desenvolvido utilizando CG

*e-mail: eliane@iq.ufrj.br acoplada a um injetor em fluxo. ${ }^{6}$ Neste método, a determinação é feita sem extração e derivatização, tendo um limite de detecção de aproximadamente $0,02 \% \mathrm{~m} / \mathrm{m}$, que parece ser insuficiente para o controle de qualidade do biodiesel, que contém concentrações menores de glicerol. Uma etapa de concentração usando extração de glicerol com água e separação cromatográfica em uma coluna capilar carbowax conduziu a um limite de detecção de aproximadamente $0,002 \% \mathrm{~m} / \mathrm{m} .{ }^{6}$ A maioria dos trabalhos sobre a utilização de CG no controle de qualidade do glicerol em amostras de biodiesel emprega a análise por injeção em fluxo..$^{7-9}$ No entanto, existem trabalhos com CG acoplada a um espectrômetro de massas. ${ }^{10}$

Outra técnica utilizada para análise de GL é a espectrofotometria na região do ultravioleta-visível (UV-Vis). O glicerol, neste caso, após a sua extração, é oxidado a formaldeído com metaperiodato de sódio, seguido da reação Hantzsch com acetilacetona, formando o composto 3,5-diacetil-1,4-di-hidrolutidina, que apresenta absorvância máxima em $410 \mathrm{~nm}^{11}$

Um método utilizando a eletroforese capilar (EC) foi desenvolvido para a quantificação de GL em diferentes amostras de biodiesel. O procedimento consistiu em, antes da análise, reagir o glicerol, inicialmente extraído da amostra de biodiesel, com periodato de sódio. ${ }^{12}$ Nessa reação, o glicerol sofre uma clivagem oxidativa produzindo formaldeído, ácido fórmico, água e iodato de sódio. O teor de glicerol é obtido indiretamente, determinando-se a concentração de iodato formado nesta reação por EC.

O teor de GT foi determinado por meio de saponificação de biodiesel com posterior determinação de glicerol por análise fotométrica utilizando enzimas. ${ }^{13} \mathrm{O}$ método, entretanto, mostrou baixa precisão, que pode estar associada ao processo de saponificação do biodiesel.

Com um procedimento parecido, a Sigma-Aldrich desenvolveu um kit (BQP-02), baseado no método Greenhill descrito numa patente, para a determinação enzimática de GL e GT em biodiesel. ${ }^{14} \mathrm{O}$ método baseia-se num procedimento fotoenzimático, semelhante ao usado nas análises de triglicerídeos no sangue, usando duas enzimas na leitura de GL e contém uma etapa de saponificação para a determinação de GT. Em algumas amostras, a presença de produtos voláteis (os solventes usados como aditivos) pode causar interferência na 
determinação do glicerol livre. ${ }^{11} \mathrm{O}$ método é rápido, preciso, de baixo custo, pouco complicado no manuseio e não tóxico, por não utilizar piridina, solvente usado na etapa de derivatização na determinação de glicerol por CG. As reações enzimáticas envolvidas neste método podem ser vistas na Figura 1.

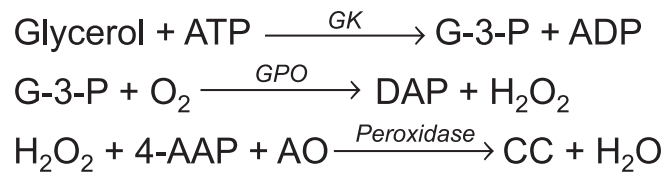

Figura 1. Reações enzimáticas envolvidas na conversão do glicerol em dihidroxiacetonafosfato pela ação sequencial das enzimas glicerol-quinase e glicerol-3-fosfato oxidase

O glicerol, na presença da adenosina trifosfato (ATP) e da enzima glicerol-quinase (GK), produz adenosina difosfato (ADP) e glicerol3-fosfato (G-3-P) (reação 1) que, na presença de glicerol-3-fosfato oxidase (GPO) e oxigênio, produz di-hidroxiacetonafosfato (DAP) e peróxido de hidrogênio $\left(\mathrm{H}_{2} \mathrm{O}_{2}\right)$ (reação 2). Finalmente, na presença de uma peroxidase, um aceptor de oxigênio $(\mathrm{AO})$ e a 4-aminoantipirina (4-AAP), são formados a água e um composto colorido (CC) (reação 3), que pode ser determinado espectrofotometricamente.

É possível, também, determinar glicerol livre e total em biodiesel a partir do consumo de oxigênio produzido durante a reação enzimática em uma célula eletroquímica, conforme indicado nas reações 1 e 2, utilizando-se para isso a célula de Clark, a qual gera uma corrente catódica que é proporcional à concentração de oxigênio presente na solução. ${ }^{2}$

A determinação de glicerol pode ainda ser feita a partir da análise amperométrica do $\mathrm{H}_{2} \mathrm{O}_{2}$ produzido na reação 2 utilizando, neste caso, um eletrodo de trabalho de platina polarizado em $+0,65 \mathrm{~V}_{\mathrm{Ag} / \mathrm{AgCl}} \mathrm{de}$ modo a oxidar o peróxido formado a oxigênio molecular. ${ }^{15}$ Este tipo de análise também foi realizado para determinação de triglicerídeo, utilizando-se um biossensor amperométrico construído a partir de uma membrana de acetato de celulose na qual foram imobilizadas três enzimas: uma lipase comercial, a GK e a GPO, sobre o eletrodo de trabalho de platina e, também, para análise de glicerol livre e total em amostras de biodiesel. ${ }^{16,17}$

Este trabalho teve como objetivo desenvolver um método analítico de baixo custo, sensível, rápido e com instrumentação simples para a determinação do teor de GL e GT em amostras de biodiesel. Para isso, foi utilizado um kit enzimático comercial para determinação de triglicerídeos com detecção colorimétrica. Pretende-se que este método atenda às especificações de qualidade da ANP, no intuito de substituir o método vigente que utiliza $\mathrm{CG}$, devido principalmente ao seu alto custo.

\section{PARTE EXPERIMENTAL}

Todas as amostras de biodiesel foram previamente analisadas por CG, no CENPES/PETROBRAS, para obtenção dos valores de referência seguindo o procedimento da norma ASTM D6584..$^{18}$ Neste método, a amostra é analisada por cromatografia a gás com detecção por ionização em chama após a sinalização com $N$-metil- $N$-(trimetilsilil)trifluoroacetamida (MSTFA). A separação cromatográfica é realizada através de uma coluna tubular aberta ( $5 \%$ fenil-polidimetilsiloxano) com 10 ou $15 \mathrm{~m}$ de comprimento, 0,32 $\mathrm{mm}$ de diâmetro interno e $0,1 \mu \mathrm{m}$ de espessura do filme. Hélio ou hidrogênio pode ser utilizado como gás de arraste, com fluxo de $3 \mathrm{~mL} / \mathrm{min}$. Uma alíquota de $1 \mu \mathrm{L}$ de amostra é injetada na coluna que é então aquecida a $50^{\circ} \mathrm{C}$. A seguinte programação do forno foi utilizada: temperatura inicial de 50 ${ }^{\circ} \mathrm{C}$ (1 min), seguida de aumento de $15^{\circ} \mathrm{C} / \mathrm{min}$ até $180^{\circ} \mathrm{C}$, e posterior aumento da temperatura de $7{ }^{\circ} \mathrm{C} / \mathrm{min}$ até $230{ }^{\circ} \mathrm{C}$ e de $30^{\circ} \mathrm{C} / \mathrm{min}$ até $380^{\circ} \mathrm{C}$. A temperatura final permaneceu por $10 \mathrm{~min}$. A temperatura do detector foi de $380{ }^{\circ} \mathrm{C}$. Para a calibração foram utilizados dois padrões internos: 1,2,4-butanotriol para glicerol e tricaprina para mono, di e triglicerídeos, além de glicerol, monooleína, dioleína e trioleína como padrões para calibração externa.

No método enzimático com detecção colorimétrica, o teor de GL foi obtido realizando-se primeiro a sua extração a partir de $400 \mu \mathrm{L}$ de biodiesel em uma mistura de $800 \mu \mathrm{L}$ de etanol absoluto, $800 \mu \mathrm{L}$ de água destilada e $1600 \mu \mathrm{L}$ de heptano. Esta mistura foi agitada em vortex por $1 \mathrm{~min}$, obteve-se a separação das fases em centrífuga por 2 min e a fase mais densa foi analisada. ${ }^{2}$

Para análise do teor de GT, uma etapa de transesterificação foi realizada no intuito de liberar todo glicerol ainda combinado nas moléculas de mono, di e triglicerídeos presentes na amostra de biodiesel. Para isso, 0,50 g de biodiesel e $2 \mathrm{~mL}$ de uma solução de $\mathrm{KOH}$ a $3 \%$ em etanol absoluto foram adicionados em um tubo com agitação e aquecimento a $65^{\circ} \mathrm{C}$ por $30 \mathrm{~min}$. Uma alíquota de $70 \mu \mathrm{L}$ de ácido acético glacial foi adicionada no intuito de neutralizar a amostra. Esta mistura foi agitada em vortex por $1 \mathrm{~min}$, sendo o glicerol então extraído com 2,0 mL de água destilada e $2 \mathrm{~mL}$ de heptano, com posterior agitação em vortex e centrifugação para separação de fases por 2 min. ${ }^{2}$ A fase mais densa contendo o glicerol total foi analisada por colorimetria. Neste trabalho, o método de transesterificação e de extração do glicerol livre seguiu o mesmo procedimento desenvolvido em trabalhos anteriores. ${ }^{2,17}$

O método enzimático com detecção colorimétrica utilizado em todas as análises baseou-se na dosagem do substrato pelo método de ponto final. A análise colorimétrica foi realizada em $505 \mathrm{~nm}$, sendo que um volume de $30 \mu \mathrm{L}$ (para GL) ou $10 \mu \mathrm{L}$ (para GT) do extrato aquoso e $1 \mathrm{~mL}$ do kit enzimático foram adicionados à cubeta de análise.

Utilizou-se um kit enzimático comercial, fornecido pela Bioclin (Quibasa), que inclui ATP a 0,250 $\mathrm{mmol} \mathrm{L}^{-1}$, cloreto de magnésio a 15 mmol L-1, GK >1500 U/L e GPO>4000 U/L, tampão PIPES pH 7,0 (100 $\left.\mathrm{mmol} \mathrm{L}^{-1}\right)$, entre outros reagentes. Heptano e etanol absoluto foram obtidos da Merck.

O efeito da temperatura na cinética das reações enzimáticas foi avaliado realizando-se análises colorimétricas com $1 \mathrm{~mL}$ de kit enzimático contendo $0,02 \mathrm{mmol} \mathrm{L}^{-1}$ de glicerol em três temperaturas diferentes: 25,37 e $50{ }^{\circ} \mathrm{C}$.

As curvas de calibração para leituras de glicerol livre e total foram obtidas utilizando-se solução padrão de glicerol em faixas de concentração de $0,005 \mathrm{mmol} \mathrm{L}^{-1}\left(4,95 \times 10^{-5} \% \mathrm{~m} / \mathrm{m}\right)$ a $0,038 \mathrm{mmol}$ $\mathrm{L}^{-1}\left(3,96 \times 10^{-4} \% \mathrm{~m} / \mathrm{m}\right)$ e $0,004 \mathrm{mmol} \mathrm{L}^{-1}\left(1,25 \times 10^{-3} \% \mathrm{~m} / \mathrm{m}\right)$ a 0,145 mmol L-1 $\left(5,33 \times 10^{-3} \% \mathrm{~m} / \mathrm{m}\right)$, respectivamente, sendo cada ponto analisado em triplicata, a $25{ }^{\circ} \mathrm{C}$. Os resultados foram submetidos ao teste de Grubbs $(\mathrm{G})$ para a retirada de qualquer valor aberrante.

Para o cálculo do limite de detecção (LD) foi utilizado o critério da inclinação da reta $(L D=3 \delta / b)$, onde $\delta$ é o desvio padrão das leituras dos brancos e b é a inclinação da reta obtida a partir da curva de calibração. O limite de quantificação (LQ) foi calculado como sendo igual a três vezes o LD. ${ }^{19}$

Amostras de biodiesel foram analisadas para determinação de GL e GT em triplicata a $25^{\circ} \mathrm{C}$, sendo os resultados submetidos ao teste de Grubbs $(\mathrm{G})$, sendo que os valores rejeitados não foram utilizados nas análises estatísticas e as médias foram obtidas para fins de comparação com os valores de referência.

A validação do método incluiu o estudo da linearidade, homocedasticidade (teste Cochran), recuperação, precisão intradia e precisão interdias.

O estudo de recuperação foi realizado a partir da quantificação de GL e GT em amostras de biodiesel previamente analisadas por CG, 
onde os testes $\mathrm{F}$ e $\mathrm{t}$ foram aplicados para fins de comparação entre os dois métodos - cromatográfico e colorimétrico.

Além disso, realizou-se também o estudo de recuperação de GL e GT em amostras de biodiesel fortificadas com valores conhecidos de glicerol e de triglicerídeos, respectivamente. Para isso, uma amostra de biodiesel de soja com teor de glicerol livre abaixo de $0,01 \%(\mathrm{~m} / \mathrm{m})$ foi fortificada com 0,$02 ; 0,03 ; 0,04$ e $0,05 \%$ em massa de glicerol para análise de GL. A recuperação do método para análise de GT foi feita fortificando-se uma amostra de biodiesel de soja, que apresentava $0,068 \%$ em massa como valor original de GT, com $0,3 \%$ em massa de triglicerídeo, utilizando-se para isso uma amostra de óleo de soja.

O estudo da precisão intradia e interdias foi realizado pela análise quantitativa de triplicatas para duas amostras distintas de biodiesel em um mesmo dia e em dois dias diferentes, respectivamente.

Todas as análises colorimétricas foram feitas em um espectrofotômetro UV-Vis Lambda XLS da Perkin Elmer.

\section{RESULTADOS E DISCUSSÃO}

A análise colorimétrica também é uma técnica bastante utilizada na determinação clínica de triglicerídeos. Os kits enzimáticos utilizados neste trabalho baseiam-se na ação acoplada de 4 enzimas: lipoproteína lípase (LPL), glicerol-quinase (GK), glicerol-3-fosfato oxidase (GPO) e uma peroxidase (POD). A LPL hidrolisa os triglicerídeos, produzindo ácidos graxos e glicerol, que é convertido a dihidroxiacetona fosfato pela ação das enzimas GK e GPO, conforme as reações 1 e 2 . O peróxido de hidrogênio formado na reação 2 , na presença de 4-aminoantipirina, 4-clorofenol (aceptor de oxigênio) e POD, produz um composto colorido, cuja absorvância máxima pode ser medida em $505 \mathrm{~nm}$, conforme a reação 3. Neste trabalho a lipoproteína lipase não desempenhou o seu papel, já que foram analisados somente extratos de glicerol em mistura de água:etanol para as análises de glicerol livre e total.

O efeito da temperatura foi avaliado com o objetivo de verificar o possível aumento da velocidade da reação enzimática e consequente diminuição do tempo de análise com o aumento da temperatura. A Figura 2 apresenta os resultados obtidos para as temperaturas de 25, 37 e $50{ }^{\circ} \mathrm{C}$. O tempo necessário para alcançar o ponto final da reação foi de aproximadamente $4 \mathrm{~min}$. Observou-se que não há uma variação significativa na velocidade da reação e, portanto, no tempo de análise com a mudança de temperatura. Além disso, constatou-se uma diminuição da resposta do sistema com o aumento da temperatura (a $50^{\circ} \mathrm{C}$ ), que pode estar relacionada à desnaturação das enzimas. Como

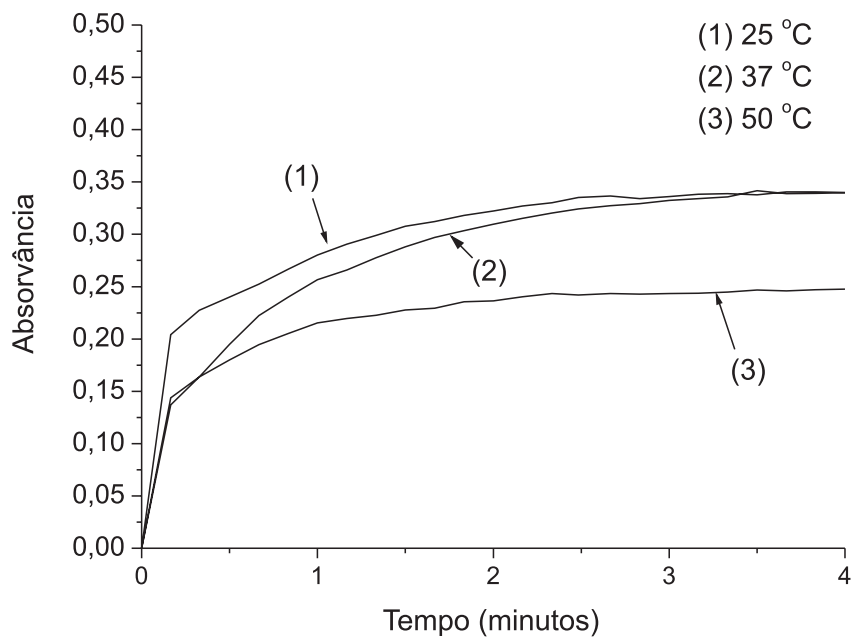

Figura 2. Efeito da temperatura no método enzimático com detecção colorimétrica nenhuma diferença significativa foi observada entre as temperaturas de 25 e $37^{\circ} \mathrm{C}$, todas as análises foram realizadas a $25^{\circ} \mathrm{C}$.

A determinação de glicerol livre e total utilizando a técnica colorimétrica foi realizada com base na linearidade do método, que foi avaliada através da construção de uma curva de calibração com glicerol, como analito, na presença do kit enzimático. O GL foi quantificado na faixa de concentração de $0,005 \mathrm{mmol} \mathrm{L}^{-1}\left(4,95 \times 10^{-5} \%\right.$ $\mathrm{m} / \mathrm{m})$ a $0,038 \mathrm{mmol} \mathrm{L}^{-1}\left(3,96 \times 10^{-4} \% \mathrm{~m} / \mathrm{m}\right)$. A curva de calibração apresentou linearidade com excelente coeficiente de correlação linear $(\mathrm{r}=0,9937)$.

Os limites de detecção e quantificação determinados pelo critério da inclinação da reta foram de $9,33 \times 10^{-6}$ e $2,80 \times 10^{-5} \% \mathrm{~m} / \mathrm{m}$, respectivamente. Já o limite de detecção obtido experimentalmente foi de $7,10 \times 10^{-6} \% \mathrm{~m} / \mathrm{m}$, equivalendo a $2,10 \times 10^{-5} \% \mathrm{~m} / \mathrm{m}$ de limite de quantificação, valores estes próximos dos calculados.

Foi observado que o valor de Cochran calculado $(0,654)$ é menor que o tabelado $(0,684)$ para se avaliar o desvio bilateral das variâncias a um nível de significância de 5\%, mostrando variâncias homogêneas, conforme o aumento da concentração. Este resultado caracteriza, portanto, um comportamento homocedástico dos dados.

Para verificar a eficiência do modelo de regressão linear proposto, foi analisado o gráfico de resíduo correspondente à curva de calibração. O resíduo é obtido pela diferença do valor previsto pela reta utilizada para a quantificação e os valores obtidos experimentalmente. Os valores obtidos para cada análise estão apresentados na Figura 3.

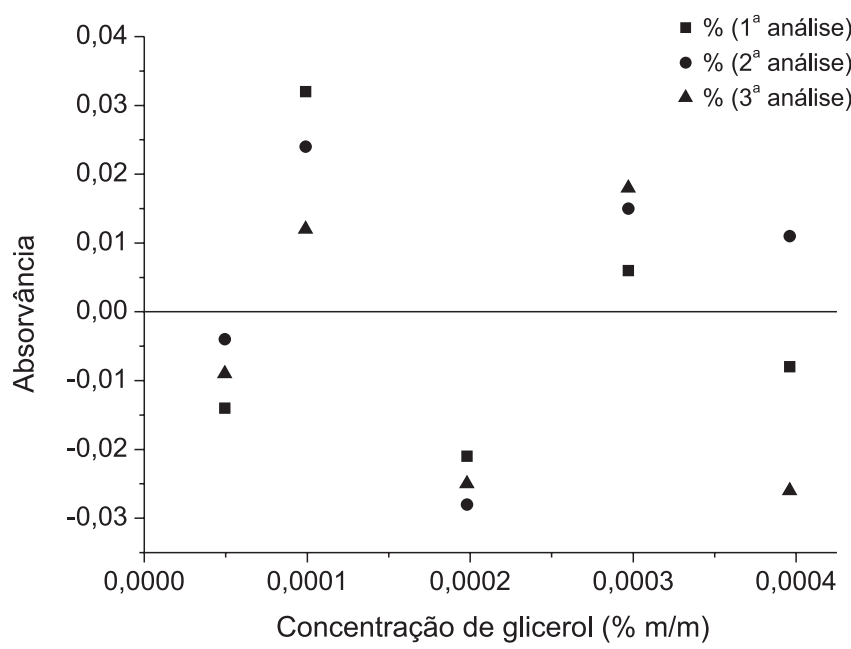

Figura 3. Gráfico de resíduos

A partir da análise do gráfico de resíduos construído foi possível avaliar que o modelo de regressão linear proposto se mostra adequado, visto que os resíduos encontrados para as análises não ultrapassaram 0,032 de absorvância, que equivale a 3,83 X 10 abaixo dos limites de detecção calculados pelo critério de inclinação da reta e obtido experimentalmente. Além disso, observa-se que o método pode ser considerado como livre de tendências, mostrando um comportamento homocedástico dos dados, corroborando o resultado obtido pelo teste de Cochran.

Pode-se confirmar, portanto, que este método enzimático com detecção colorimétrica pode ser usado na determinação de glicerol.

Com base nesses resultados, foram avaliadas 12 amostras de biodiesel, no intuito de quantificar colorimetricamente o teor de GL. Os valores médios obtidos podem ser vistos na Tabela 1 junto com o desvio padrão para cada amostra. Para efeito de comparação, os valores obtidos por CG também são apresentados, além da recuperação do método e o erro relativo ao valor obtido por CG. 
Tabela 1. Valores obtidos pelos métodos enzimático com detecção colorimétrica e cromatográfico, para quantificação de glicerol livre em diferentes amostras de biodiesel

\begin{tabular}{|c|c|c|c|c|c|c|}
\hline Amostra & $\begin{array}{c}\text { Valores obtidos } \\
(\% \mathrm{~m} / \mathrm{m})\end{array}$ & $\begin{array}{c}\text { Média } \\
(\% \mathrm{~m} / \mathrm{m})\end{array}$ & $\mathrm{s}$ & $\begin{array}{c}\mathrm{CG} \\
(\% \mathrm{~m} / \mathrm{m}) \\
\end{array}$ & $\begin{array}{c}\text { Recuperação } \\
(\%)\end{array}$ & $\begin{array}{l}\text { Erro } \\
(\%)\end{array}$ \\
\hline 1 & $\begin{array}{l}0,0053 \\
0,0048 \\
0,0052\end{array}$ & 0,0051 & 0,0003 & 0,0050 & 102 & 2 \\
\hline 2 & $\begin{array}{l}0,0071 \\
0,0077 \\
0,0092\end{array}$ & 0,0080 & 0,0011 & 0,0080 & 100 & 0 \\
\hline 3 & $\begin{array}{l}0,0084 \\
0,0069 \\
0,0086\end{array}$ & 0,0080 & 0,0009 & 0,0080 & 100 & 0 \\
\hline 4 & $\begin{array}{l}0,0154 \\
0,0153 \\
0,0151\end{array}$ & 0,0153 & 0,0002 & 0,0140 & 109 & 9 \\
\hline 5 & $\begin{array}{l}0,0123 \\
0,0134 \\
0,0136\end{array}$ & 0,0131 & 0,0007 & 0,0140 & 94 & -6 \\
\hline 6 & $\begin{array}{l}0,0107 \\
0,0107 \\
0,0118\end{array}$ & 0,0111 & 0,0006 & 0,0100 & 111 & 11 \\
\hline 7 & $\begin{array}{l}0,0099 \\
0,0098 \\
0,0098\end{array}$ & 0,0098 & 0,0001 & 0,010 & 98 & -2 \\
\hline 8 & $\begin{array}{l}0,0099 \\
0,0096 \\
0,0095\end{array}$ & 0,0097 & 0,0002 & 0,0100 & 97 & -3 \\
\hline 9 & $\begin{array}{l}0,2385 \\
0,2373 \\
0,2328\end{array}$ & 0,2362 & 0,0030 & 0,2300 & 103 & 3 \\
\hline 10 & $\begin{array}{l}0,2001 \\
0,1999 \\
0,1988\end{array}$ & 0,1996 & 0,0007 & 0,2000 & 100 & 0 \\
\hline 11 & $\begin{array}{l}0,2138 \\
0,2148 \\
0,2127\end{array}$ & 0,2138 & 0,0011 & 0,2100 & 102 & 2 \\
\hline 12 & $\begin{array}{l}0,2217 \\
0,2237 \\
0,2285 \\
\end{array}$ & 0,2246 & 0,0035 & 0,2200 & 102 & 2 \\
\hline
\end{tabular}

As amostras apresentaram recuperação de 94 a $111 \%$ e um baixo erro relativo em relação ao valor obtido por CG. Sobre a homogeneidade dos resultados de GL quantificado pelos dois métodos (cromatográfico e colorimétrico), foi observado que o valor calculado de $\mathrm{F}$ $(1,04)$ é menor que o valor de F crítico $(2,82)$ considerando $95 \%$ de confiança, podendo-se concluir que as variâncias dos dois métodos são equivalentes. Deste modo, um segundo cálculo foi feito para o valor de $\mathrm{t}$ (bilateral) para os dois métodos presumindo variâncias equivalentes. Pelos resultados, obteve-se o valor de t calculado $(0,98)$ menor que o de $t$ crítico $(2,07)$ considerando $95 \%$ de confiança, o que mostra que as médias para os dois métodos são equivalentes entre si. Ou seja, o método enzimático de determinação de glicerol livre em diferentes amostras de biodiesel com detecção colorimétrica proposto neste trabalho pode ser usado, pois apresenta resultados equivalentes ao método padrão (CG).

A Figura 4 apresenta a correlação entre os métodos enzimático com detecção colorimétrica e CG. O coeficiente angular foi de 0,9836 e o valor obtido para o coeficiente de correlação linear foi de 0,9999 , mostrando uma excelente correlação entre os resultados obtidos pelos dois métodos.

Para duas amostras distintas de biodiesel, determinou-se a precisão intradia e interdia, conforme pode ser visto na Tabela 2. Os resultados de precisão intra e interdia para a amostra 1 são 0,0150

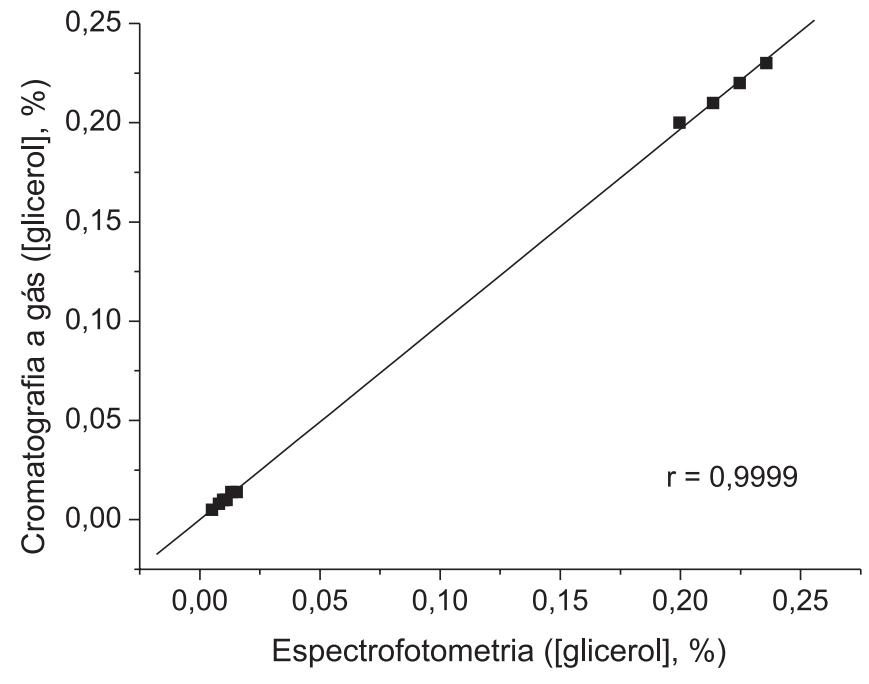

Figura 4. Correlação entre os métodos utilizados para determinação de glicerol livre: método enzimático com detecção colorimétrica e CG

$\pm 0,0003$ e $0,014 \pm 0,001$, e para a amostra 2 são $0,0095 \pm 0,0003$ e $0,0096 \pm 0,0001$, respectivamente. Não houve, portanto, variação 
Tabela 2. Resultados de glicerol livre obtidos pelo método enzimático com detecção colorimétrica no mesmo dia e em dias diferentes

\begin{tabular}{|c|c|c|c|c|c|c|}
\hline \multicolumn{7}{|c|}{$1^{\circ}$ dia } \\
\hline Amostra & $\begin{array}{l}\text { Valores obtidos } \\
\qquad \% \mathrm{~m} / \mathrm{m})\end{array}$ & $\begin{array}{c}\text { Média } \\
(\% \mathrm{~m} / \mathrm{m})\end{array}$ & $\mathrm{s}$ & $\begin{array}{c}\mathrm{CG} \\
(\% \mathrm{~m} / \mathrm{m})\end{array}$ & $\begin{array}{c}\text { Recuperação } \\
(\%)\end{array}$ & $\begin{array}{r}\text { Erro } \\
(\%)\end{array}$ \\
\hline 1 & $\begin{array}{l}0,0143 \\
0,0135 \\
0,0136\end{array}$ & 0,0138 & 0,0004 & 0,0140 & 99 & -1 \\
\hline 2 & $\begin{array}{l}0,0098 \\
0,0095 \\
0,0093 \\
\end{array}$ & 0,0095 & 0,0003 & 0,0100 & 95 & -5 \\
\hline \multicolumn{7}{|c|}{$2^{o}$ dia } \\
\hline Amostra & $\begin{array}{l}\text { Valores obtidos }(\% \\
\mathrm{m} / \mathrm{m})\end{array}$ & $\begin{array}{c}\text { Média } \\
(\% \mathrm{~m} / \mathrm{m})\end{array}$ & $\mathrm{s}$ & $\begin{array}{c}\mathrm{CG} \\
(\% \mathrm{~m} / \mathrm{m})\end{array}$ & $\begin{array}{c}\text { Recuperação } \\
(\%)\end{array}$ & $\begin{array}{l}\text { Erro } \\
(\%)\end{array}$ \\
\hline 1 & $\begin{array}{l}0,0123 \\
0,0134 \\
0,0136\end{array}$ & 0,0131 & 0,0007 & 0,0140 & 94 & -6 \\
\hline 2 & $\begin{array}{l}0,0099 \\
0,0096 \\
0,0095 \\
\end{array}$ & 0,0097 & 0,0002 & 0,010 & 97 & -3 \\
\hline
\end{tabular}

significativa dos resultados obtidos no mesmo dia e em dias diferentes, mostrando que o método enzimático colorimétrico é preciso na análise de glicerol livre em amostras de biodiesel.

A Tabela 3 apresenta os resultados obtidos de recuperação em amostras de biodiesel fortificadas com valores conhecidos de glicerol. Observa-se nesta tabela bons resultados de recuperação, variando de 105 a 108\%, sendo possível concluir que o procedimento de extração do glicerol livre proposto em trabalhos anteriores pode ser utilizado como método padrão na quantificação de glicerol livre em amostras de biodiesel. ${ }^{2,17}$

A Tabela 4 apresenta os valores médios obtidos de glicerol total junto com o desvio padrão das amostras, assim como os valores obtidos por CG, a recuperação do método e o erro relativo da amostra.

Os resultados do teor de glicerol total determinado pelo método enzimático colorimétrico apresentaram recuperação na faixa de 80 a $119 \%$ e um baixo erro relativo em relação ao valor obtido por CG. Quanto à homogeneidade dos resultados de glicerol total quantificado pelos métodos cromatográfico e colorimétrico, verificou-se
Tabela 3. Resultados obtidos pelo método enzimático com detecção colorimétrica para a amostra de biodiesel de soja fortificada com teor de glicerol livre de $0,03,0,04$ e $0,05 \%(\mathrm{~m} / \mathrm{m})$

\begin{tabular}{lccc}
\hline Amostra & $\begin{array}{c}\text { Valores obtidos } \\
(\% \mathrm{~m} / \mathrm{m})\end{array}$ & $\begin{array}{c}\text { Média } \\
(\% \mathrm{~m} / \mathrm{m})\end{array}$ & $\begin{array}{c}\text { Recuperação } \\
(\%)\end{array}$ \\
\hline Biodiesel puro & $<0,0100$ & $<0,0100$ & - \\
Fortificada com $0,02 \% \mathrm{~m} / \mathrm{m}$ & 0,0206 & 0,0210 & 105 \\
& 0,0211 & & \\
& 0,0212 & & \\
Fortificada com $0,03 \% \mathrm{~m} / \mathrm{m}$ & 0,0306 & 0,0316 & 105 \\
& 0,0318 & & \\
& 0,0323 & & \\
Fortificada com $0,04 \% \mathrm{~m} / \mathrm{m}$ & 0,0427 & 0,0432 & 108 \\
& 0,0430 & & \\
& 0,0438 & & \\
Fortificada com $0,05 \% \mathrm{~m} / \mathrm{m}$ & 0,0516 & 0,0530 & \\
& 0,0531 & & \\
\hline
\end{tabular}

Tabela 4. Valores obtidos pelos métodos enzimático com detecção colorimétrica e cromatográfico, para quantificação de glicerol total em diferentes amostras de biodiesel

\begin{tabular}{|c|c|c|c|c|c|c|}
\hline Amostra & $\begin{array}{c}\text { Valores obtidos } \\
(\% \mathrm{~m} / \mathrm{m})\end{array}$ & $\begin{array}{c}\text { Média } \\
(\% \mathrm{~m} / \mathrm{m})\end{array}$ & $\mathrm{s}$ & $\begin{array}{c}\mathrm{CG} \\
(\% \mathrm{~m} / \mathrm{m}) \\
\end{array}$ & $\begin{array}{c}\text { Recuperação } \\
(\%)\end{array}$ & $\begin{array}{l}\text { Erro } \\
(\%) \\
\end{array}$ \\
\hline 1 & $\begin{array}{l}0,5364 \\
0,5562\end{array}$ & 0,5463 & 0,0140 & 0,4600 & 119 & 19 \\
\hline 2 & $\begin{array}{l}0,2632 \\
0,2486\end{array}$ & 0,2559 & 0,0103 & 0,2550 & 100 & 0 \\
\hline 3 & $\begin{array}{l}0,2677 \\
0,2745\end{array}$ & 0,2711 & 0,0048 & 0,3280 & 83 & -17 \\
\hline 4 & $\begin{array}{l}0,4173 \\
0,4050\end{array}$ & 0,4112 & 0,0087 & 0,4140 & 99 & -1 \\
\hline 5 & $\begin{array}{l}0,1894 \\
0,1878\end{array}$ & 0,1886 & 0,0012 & 0,2030 & 93 & -7 \\
\hline 6 & $\begin{array}{l}0,0970 \\
0,1130\end{array}$ & 0,1050 & 0,0113 & 0,1050 & 100 & 0 \\
\hline 7 & $\begin{array}{l}0,1621 \\
0,1574\end{array}$ & 0,1597 & 0,0033 & 0,1780 & 90 & -10 \\
\hline 8 & $\begin{array}{l}0,1677 \\
0,1698\end{array}$ & 0,1688 & 0,0015 & 0,1710 & 99 & -1 \\
\hline 9 & $\begin{array}{l}0,1725 \\
0,1879\end{array}$ & 0,1802 & 0,0109 & 0,2260 & 80 & -20 \\
\hline 10 & $\begin{array}{l}0,3482 \\
0,3499\end{array}$ & 0,3491 & 0,0012 & 0,3760 & 93 & -7 \\
\hline
\end{tabular}


Tabela 5. Resultados de glicerol total obtidos pelo método enzimático com detecção colorimétrica no mesmo dia e em dias diferentes

\begin{tabular}{|c|c|c|c|c|c|c|}
\hline \multicolumn{7}{|c|}{$1^{\circ} \mathrm{dia}$} \\
\hline Amostra & $\begin{array}{l}\text { Valores obtidos } \\
(\% \mathrm{~m} / \mathrm{m})\end{array}$ & $\begin{array}{c}\text { Média } \\
(\% \mathrm{~m} / \mathrm{m})\end{array}$ & $\mathrm{s}$ & $\begin{array}{c}\mathrm{CG} \\
(\% \mathrm{~m} / \mathrm{m})\end{array}$ & $\begin{array}{c}\text { Recuperação } \\
(\%)\end{array}$ & $\begin{array}{l}\text { Erro } \\
(\%)\end{array}$ \\
\hline 1 & $\begin{array}{l}0,2899 \\
0,2769 \\
0,2672\end{array}$ & 0,2780 & 0,0114 & 0,3000 & 93 & -7 \\
\hline 2 & $\begin{array}{l}0,1366 \\
0,1475 \\
0,1310\end{array}$ & 0,1384 & 0,0084 & 0,1300 & 106 & 6 \\
\hline \multicolumn{7}{|c|}{$2^{\circ}$ dia } \\
\hline Amostra & $\begin{array}{l}\text { Valores obtidos } \\
\qquad(\% \mathrm{~m} / \mathrm{m})\end{array}$ & $\begin{array}{l}\text { Média } \\
(\% \mathrm{~m} / \mathrm{m})\end{array}$ & $\mathrm{s}$ & $\begin{array}{c}\mathrm{CG} \\
(\% \mathrm{~m} / \mathrm{m})\end{array}$ & $\begin{array}{c}\text { Recuperação } \\
(\%)\end{array}$ & $\begin{array}{l}\text { Erro } \\
(\%)\end{array}$ \\
\hline 1 & $\begin{array}{l}0,2986 \\
0,2899 \\
0,2961\end{array}$ & 0,2949 & 0,0045 & 0,3000 & 98 & -2 \\
\hline 2 & $\begin{array}{l}0,1272 \\
0,1281 \\
0,1310\end{array}$ & 0,1288 & 0,0020 & 0,1300 & 99 & -1 \\
\hline
\end{tabular}

que o valor calculado de $\mathrm{F}(1,35)$ é menor que o valor de $\mathrm{F}$ crítico $(3,18)$ considerando $95 \%$ de confiança, podendo-se concluir que as variâncias dos dois métodos são iguais. Deste modo, foi realizado um segundo cálculo para o valor de t (bilateral) para os dois métodos, presumindo-se variâncias iguais. Pelos resultados, foi verificado que o valor de t calculado $(0,889)$ é menor que o de t crítico $(2,101)$ considerando $95 \%$ de confiança, podendo-se afirmar que as médias para os dois métodos são equivalentes entre si. Ou seja, o método proposto neste trabalho pode ser usado na determinação de glicerol total em amostras de biodiesel, pois apresenta resultados equivalentes ao método padrão (CG).

Na Figura 5 é possível ver a correlação entre os resultados obtidos pelos métodos: enzimático colorimétrico e CG, apresentados na Tabela 4. O coeficiente angular da reta foi de 0,8304 e o valor obtido de coeficiente de correlação linear foi de 0,9645, mostrando uma razoável correlação entre os resultados obtidos pelos dois métodos.

Também para a análise de glicerol total, determinou-se a precisão intradia e interdia, conforme apresentado na Tabela 5. Os resultados de precisão intradia e interdias para a amostra 1 são $0,28 \pm 0,01 \mathrm{e}$ $0,29 \pm 0,01$, e para a amostra 2 são $0,138 \pm 0,008$ e $0,134 \pm 0,007$,

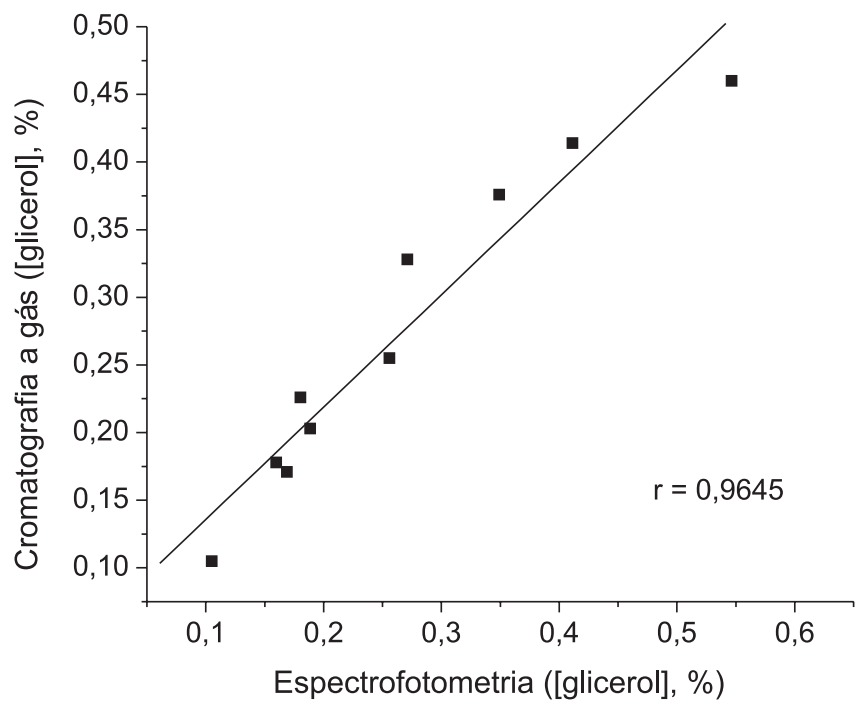

Figura 5. Correlação entre os métodos utilizados para determinação de glicerol total: método enzimático com detecção colorimétrica e CG respectivamente. Não houve variação significativa dos resultados obtidos no mesmo dia e em dias diferentes, mostrando que o método enzimático colorimétrico envolvendo a reação de transesterificação também é preciso na análise de glicerol total em amostras de biodiesel.

A Tabela 6 apresenta os valores de recuperação de GT em uma amostra de biodiesel de soja fortificada com teor de triglicerídeo de $0,3 \%(\mathrm{~m} / \mathrm{m})$. Observa-se nesta tabela excelente recuperação com um valor médio de $101 \%$.

Tabela 6. Média dos resultados obtidos pelo método enzimático com detecção colorimétrica para amostra de biodiesel de soja fortificada com teor de triglicerídeo de $0,3 \%(\mathrm{~m} / \mathrm{m})$

\begin{tabular}{lccc}
\hline Amostra & $\begin{array}{c}\text { Valores obtidos } \\
(\% \mathrm{~m} / \mathrm{m})\end{array}$ & $\begin{array}{c}\text { Média } \\
(\% \mathrm{~m} / \mathrm{m})\end{array}$ & $\begin{array}{c}\text { Recuperação } \\
(\%)\end{array}$ \\
\hline Biodiesel puro & 0,0598 & 0,0610 & 90 \\
& 0,0602 & & \\
& 0,0630 & & \\
Fortificada com $0,3 \% \mathrm{~m} / \mathrm{m}$ & $0,3083^{*}$ & 0,3032 & 101 \\
& $0,2996^{*}$ & & \\
& $0,3016^{*}$ & & \\
\hline
\end{tabular}

*Valores já descontados da média dos valores obtidos para o branco

\section{CONCLUSÕES}

O método enzimático colorimétrico utilizando-se kits comerciais para análise de triglicerídeos é simples, rápido e viável na determinação do teor de glicerol livre e total em amostras de biodiesel.

O método mostrou ser linear, apresenta comportamento homocedástico e valores de limites de detecção e de quantificação de $7,10 \times 10^{-6}$ e $2,10 \times 10^{-5} \% \mathrm{~m} / \mathrm{m}$, respectivamente, determinados pelo método experimental.

A análise de glicerol livre foi precedida de uma etapa de extração do glicerol em uma mistura de água:etanol e mostrou correlação de 98\% com os resultados obtidos pela técnica de cromatografia a gás, além de boa precisão intradia e interdia e recuperações variando de 105 a $108 \%$, quando amostras foram fortificadas com diferentes concentrações de glicerol.

A análise de glicerol total foi precedida de uma reação de transesterificação e de uma etapa de extração do glicerol livre e mostrou correlação de $83 \%$ com os resultados obtidos pela técnica de cromatografia a gás, além de boa precisão intradia e interdia e excelente 
recuperação (101\%), quando uma amostra de biodiesel foi fortificada com óleo de soja.

\section{AGRADECIMENTOS}

Ao CENPES/PETROBRAS e CNPq pelo apoio financeiro e ao CNPq e CAPES pelas bolsas de pesquisa concedidas.

\section{REFERENCIAS}

1. Rocha, D. Q.; Barros, D. K.; Costa, E. J. C.; Souza, K. S.; Passos, R. R.; Veiga Jr., V. F.; Chaar, J. S.; Quim. Nova 2008, 31, 1062.

2. Luetkmeyer, T.; Santos, R. M.; Silva, A. B.; Amado, R. S.; Vieira, E. C.; D'Elia, E.; Electroanalysis 2010, 22, 995.

3. Monteiro, M. R.; Ambrozin, A. R. P.; Lião, L. M.; Ferreira, A. G.; Talanta 2008, 77, 593.

4. http://nxt.anp.gov.br/nxt/gateway.dll/leg/resolucoes_anp/2010/fevereiro/ ranp\% 204\% 20-\%202010.xml?f=templates $\$ \mathrm{fn}=$ document-frame htm $\$ 3.0 \$ q=\$ x$, acessada em Agosto 2011.

5. Freedman, B.; Kwolek, W. F.; Pryde, E. H.; J. Am. Oil Chem. Soc. 1986, $63,1370$.

6. Bondioli, P.; Mariani, C.; Lanzani, A.; Fedeli, E.; Veronese, S.; Riv. Ital. Sostanze Grasse 1992, 7, 7.
7. Knothe, G.; J. Am. Oil Chem. Soc. 2006, 83, 823 .

8. Mittelbach, M.; Chromatographia 1993, 37, 623.

9. Mittelbach, M; Roth, G.; Bergmann, A.; Chromatographia 1996, 42, 431.

10. Plank, C.; Lorbeer, E.; J. High Res. Chromatogr. 2005, 15, 609.

11. Bondioli, P.; Bella, L. D.; Eur. J. Lipid Sci. Technol. 2005, 107, 153.

12. Gonçalves, L. C. F.; Micke, G. A.; J. Chromatogr., A 2007, 1154, 477

13. Bailer, J.; De Hueber, K.; Fresenius J. Anal. Chem. 1991, 340, 186.

14. Greenhill, S. J.; US pat. 0137546 A1 2004.

15. Wu, L. C. ; Cheng, C. M.; Anal. Biochem. 2005, 346, 234.

16. Minakshi, C. S.; Pundir; Sens. Actuators, B 2008, 133, 251.

17. Pegas, M. M.; Amado, R. S.; Castro, E. V.; D’Elia, E.; J. Appl. Electrochem. 2010, 40, 2061.

18. ASTM, American Society for Testing and Material, ASTM Committee of Standards, West Conshohocken; Standard Test Method for Determination of Total Monoglycerides, Total Diglycerides, Total Triglycerides, and Free and Total Glycerin in B-100 Biodiesel Methyl Esters by Gas Chromatography; ASTM D 6584, 2011.

19. Brett, C. M. A.; Brett, A. M. O.; Electroanalysis, Oxford University Press Inc.: New York, 1998. 